tions always play a decisive role in theoretical physics.

With very few exceptions, the exact solutions of many-body problems can be found only in one dimension. For example, the Tomonaga-Luttinger solution for a onedimensional system of interacting fermions (particles such as electrons) demonstrates how the Landau-Fermi liquid description of electrons, which has been the theoretical basis of the whole quantum theory of solids, breaks down in one or two dimensions. This discovery had a considerable impact on condensed-matter physics, and gave rise to a new field concerned with the properties of the one-dimensional state known as the 'Luttinger liquid'. In contrast, several exactly solvable models have been developed in nuclear physics that make use of grouptheory techniques. These models are based on the concept of dynamical symmetries, and a non-trivial generalization of this approach is the 'pairing model', in which particles that form a pair interact through a force with a particular strength. Such a model has been applied to discussions of superfluidity, and also to studies of superconductivity in small metallic grains.

The pairing model was solved exactly by Richardson ${ }^{2}$ more than 30 years ago, a result that passed almost unnoticed despite its enormous potential impact on nuclear as well as condensed-matter physics. In their paper, Dukelsky and colleagues ${ }^{1}$ provide an important generalization of Richardson's work, leading to new classes of pairing models that are exactly solvable in any dimension. To obtain this result, the authors apply group-theoretical and algebraic methods similar to those already used by Gaudin ${ }^{3}$. They discover three classes of integrable (a remarkable property that makes them mathematically tractable) pairing models, which they were able to solve exactly.

In analogy with the work of Gaudin, the three new classes of pairing models found by Dukelsky et al. ${ }^{1}$ are named the rational, the trigonometric and the hyperbolic models. The authors go on to apply the rational model to a two-dimensional system of 18 fermions with repulsive interactions, for which they find an exact numerical solution. Their first results indicate that there are attractive correlations between pairs of fermions, despite the underlying repulsive interaction. Such models might apply to high-temperature superconductivity, in which mutually repulsive electrons have to pair up to flow without resistance. In further work, Dukelsky et al. ${ }^{4}$ propose an exact correspondence between the solution of the quantum pairing model and a twodimensional classical electrostatic problem: the trigonometric model can be mapped to an electrostatic problem on a spherical surface, whereas the hyperbolic model seems to correspond to a mapping on a more general surface.

The new solutions of the pairing models seem to be limited to systems of finite size a necessary condition for solving equations giving exact quantum states. But they can certainly be applied to systems where $n$ is quite large, from which the extrapolation to infinite $n$ is fairly accurate. The solutions are also restricted to systems at a temperature of absolute zero $\left(-273^{\circ} \mathrm{C}\right)$, but because the exact solution provides all the quantum states and their energies, any thermodynamic quantity can be calculated at a non-zero temperature by thermal averaging, according to the Boltzmann formula of statistical mechanics. Exact solutions, as well as predicting the particular properties of a simplified system, can also indicate how good a given approximation is to a many-body system.

In all these respects, the models of Dukelsky et al. are highly welcome. Another useful feature of these models is that they apply equally to boson and fermion systems. All known particles are either fermions (such as electrons) or bosons (such as photons), which obey different quantum statistics. As the authors show ${ }^{1}$, the rational model may have some bearing on fermion systems, in particular the physics of high-temperature superconductors. In the case of boson systems, Dukelsky and Shuck ${ }^{5}$ have applied the rational model to the transition of a group of low-temperature atoms to a Bose-Einstein condensate - a state of matter in which all the atoms share the same quantum state. In similar work, Dukelsky and Pittel ${ }^{6}$ applied the model to a system of bosons with shortrange repulsive interactions, and obtained new evidence for the validity of the 'interacting boson model' of nuclear physics.

The physics of strongly correlated fermion systems is one area of active research that should benefit immediately from these new families of exactly solvable models. Despite considerable theoretical efforts, mainly motivated by the problems raised by high-temperature superconductivity, such systems remain a huge challenge to theorists. These, and other seemingly intractable problems, would welcome any insight offered by a new family of theoretical tools. The results obtained by Dukelsky et al. ${ }^{1}$ for a two-dimensional system of fermions with repulsive interactions is a first step in this direction.

Michel Héritier is in the Laboratoire de Physique des Solides d'Orsay, Centre Scientifique d'Orsay, 91405 Orsay, France.

e-mail: heritier@lps.u-psud.fr

1. Dukelsky, J., Esebbag, C. \& Schuck, P. Phys. Rev. Lett. 87, 066403 (2001)

2. Richardson, R. W. Phys. Lett. 3, 277-279 (1963)

3. Gaudin, M. J. Phys. (Paris) 37, 1087-1098 (1976).

4. Dukelsky, J. et. al. Phys. Rev. Lett. (submitted).

Dukelsky, J. \& Schuck, P. Phys. Rev. Lett. 86, 4207-4210 (2001)

6. Dukelsky, J. \& Pittel, S. Phys. Rev. Lett. 86, 4791-4794 (2001).

\section{Recycled oil}

The internal-combustion engine, says Daedalus, is inherently dirty. A gas explosion or ignition occurs in a cold cylinder, and the molecular combustion chains are quenched on its cold walls. DREADCO engineers are now seeking to make the two-stroke diesel, the dirtiest of them all, into a clean motor.

Daedalus recalls one test diesel engine that could not be turned off because it was burning its own lubricating oil. Old technology used mixed oil and petrol to drive and lubricate a two-stroke engine. So Daedalus's new engine will use the same oil first as lubricant and then as fuel.

A diesel engine is ideal for such experiments because it can burn almost anything. Whereas petrol has to be carefully formulated, the earliest diesel burnt coal dust, and almost any oil will power a diesel somehow. The ideal engine would have a cylinder so thick with oil that all combustion chains would be terminated by a liquid film. The fastflowing lubricant would be rapidly dirtied, because all the pollution would end up in it. But instead of cleaning the oil, Daedalus wants to burn it.

The key component here is the cooling system. Every diesel engine has to have one - apart from the adiabatic diesel, that is, which just runs hot - and Daedalus proposes one large enough to cool not merely the engine block, but the exhaust gas as well. The engine will then discharge cooled filthy exhaust. Daedalus wants to flow this outgoing exhaust against incoming spent lubricating oil, possibly on a rotating disc or corrugated cylinder. If this is designed properly, the outgoing gas should yield up its nasties to the incoming oil. Carbon, other solid particulates, nitrogen oxides and similar pollutants would be taken up by the spent oil, leaving only clean air (still carrying water vapour and carbon dioxide) to leave the system. The dirty oil will feed the diesel. It will first lubricate, and then drive, the engine.

The new DREADCO engine will have a modest capacity, much less than a litre. Its cooling and auxiliary gear will be heavy, making it more suitable for urban vans than for motor cars. Its cleanliness and use of fuel as lubricant will be a bonus. But Daedalus hopes that the use of incoming fuel as a pollutionscavenger will be more widely adopted by internal-combustion engineers. It should give their engine another boost in its long fight against the encroaching fuel cell. David Jones 